\title{
Comorbidities and treatment patterns among patients with acromegaly in Sweden - a register-linkage population-based study
}

\section{Introduction}

- Acromegaly is a rare disease most commonly caused by increased growth hormone $(\mathrm{GH})$ secretion from a pituitary adenoma

Acromegaly is associated with a number of comorbidities affecting e.g. the cardiovascular system, the musculoskeletal organs and metabolism Treatment of patients with acromegaly is complex, and includes surgery, pharmacotherapy and radiation therapy

The objective was to describe comorbidities and treatment patterns among patients with acromegaly in Sweden

\section{Methods}

\section{Patients}

- Adult patients (aged $>18$ years) with a first diagnosis of acromegaly due to a pituitary adenoma in Sweden between 1 July 2005 and 31 December 2013

Design

- Observational, population-based study conducted in Sweden

Linkage of nationwide registers with high precision via the unique personal identification number

Data sources

- National Patient Register; for data on specialized health care contacts, including surgical and medical interventions

Swedish Prescribed Drug Register; for data on prescribed medicines purchased in pharmacies

- Cause of Death Register; for data on time and cause of death (for censoring purposes)

\section{Endpoints and data analyses}

- The presence of comorbidities was assessed

The following treatment types were included: surgery, radiation therapy somatostatin analogues, dopamine agonists (DA) and growth hormone receptor antagonists (GHRA)

The proportion of patients receiving each type of treatment at any time from date of diagnosis until death or the end of the study period was assessed, as well as the longitudinal treatment patterns

- The analyses were performed in the total study population as well as in subgroups based on time of diagnosis (before 2010 vs. on or after 2010) - Descriptive statistics were used

\section{Results}

\section{Patient population}

Predefined selection criteria identified 358 patients ( $48 \%$ men, mean age at diagnosis 50.0 [SD 15.3] years)

$186(52 \%)$ had received their first diagnosis before 2010, and $172(48 \%)$ had been diagnosed on or after 2010

- The mean follow-up time from diagnosis to death or 31 December 2013 was 4.2 (SD 2.5) years

The follow-up time was 6.2 (SD 1.6) years among patients diagnosed before 2010, and 2.0 (SD 1.1) years among those diagnosed on or after 2010

\section{Comorbidities}

- In the total study population, $81 \%$ (290/358) had at least one comorbidity Comorbidities were present among $88 \%(164 / 186)$ of patients diagnosed before 2010 and among $73 \%(126 / 172)$ of patients diagnosed on or after 2010

- Figure 1 presents the 10 most common comorbidities
Occurrence of each type of treatment

- The percentage of patients with each type of treatment is presented in Figure 2

- Two thirds $(66 \% ; 235 / 358)$ had undergone surgery at any time during follow-up

- 86 patients had more than one surgery

- One third $(36 \% ; 127 / 358)$ had been treated with SSA at any time during follow-up; most commonly as monotherapy (i.e. not in combination with DA or GHRA) $(33 \% ; 119 / 358)$

Longitudinal treatment patterns

- First-line treatment was initiated on average 3.7 (mean; SD 6.9) months after diagnosis

$16 \%(57 / 358)$ received no treatment for acromegaly during the follow-up period

period

301 patients who received treatment the most common firstline treatments were surgery $(n=180 ; 60 \%), S S A(n=64 ; 21 \%)$ and DA $(n=41 ; 14 \%)$

- Second-line treatments after primary surgical treatment are presented in Figure 3

Treatments following first-line SSA are presented in Figure 4

Time to treatment initiation was shorter among patients diagnosed on or after 2010 than among those diagnosed before 2010 (not shown in figure)

\section{Conclusions}

- Comorbidity in patients with acromegaly is very high

- The most common first-line treatment was surgery, with the majority not receiving any second-line treatment

- SSA was the second most common first-line treatment often followed by second-line surgery

- Time to treatment initiation was shorter among patients with a more recent diagnosis

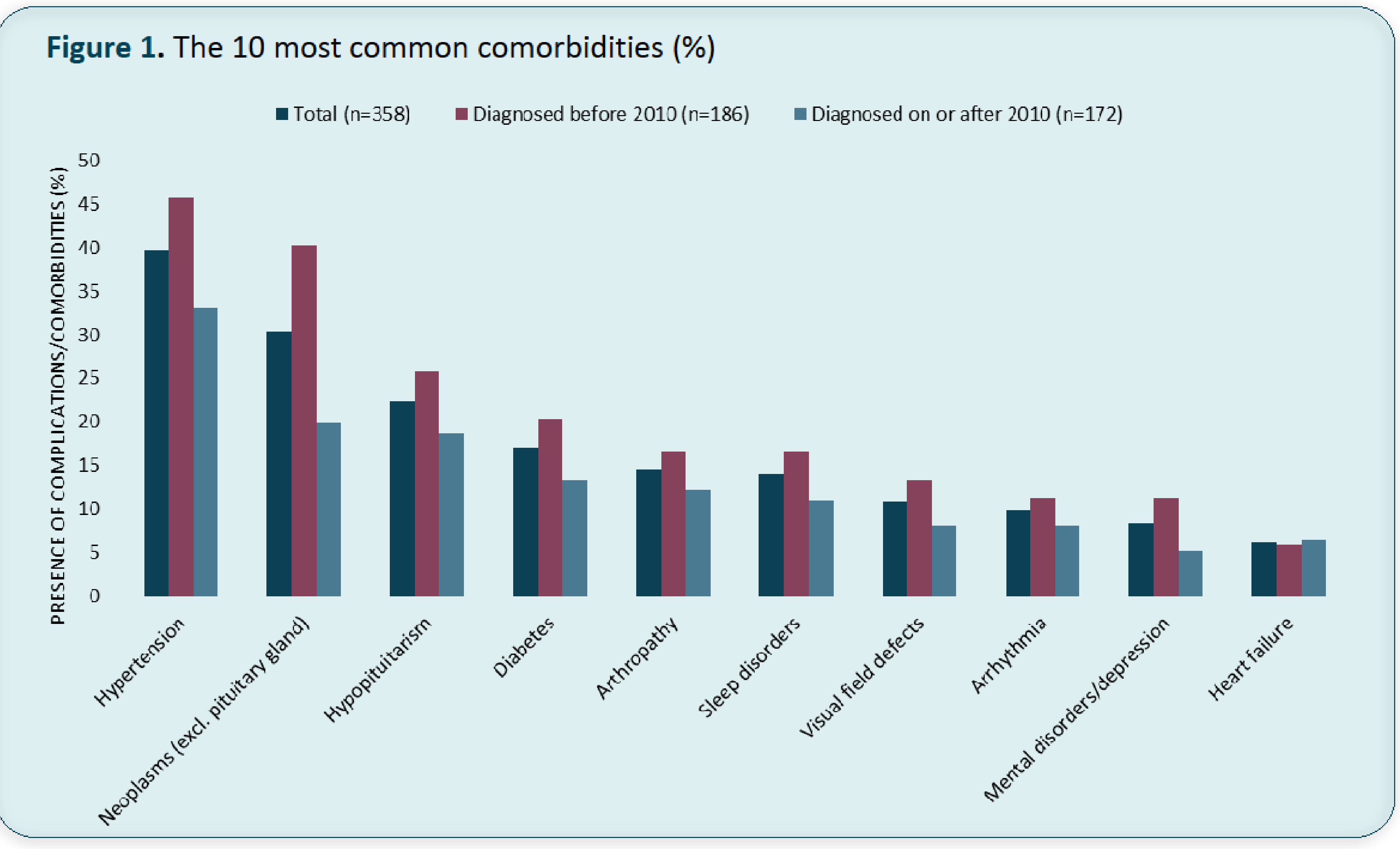

Figure 3. Second-line treatments after first-line surgery, in the total study population

\section{First-line treatment}

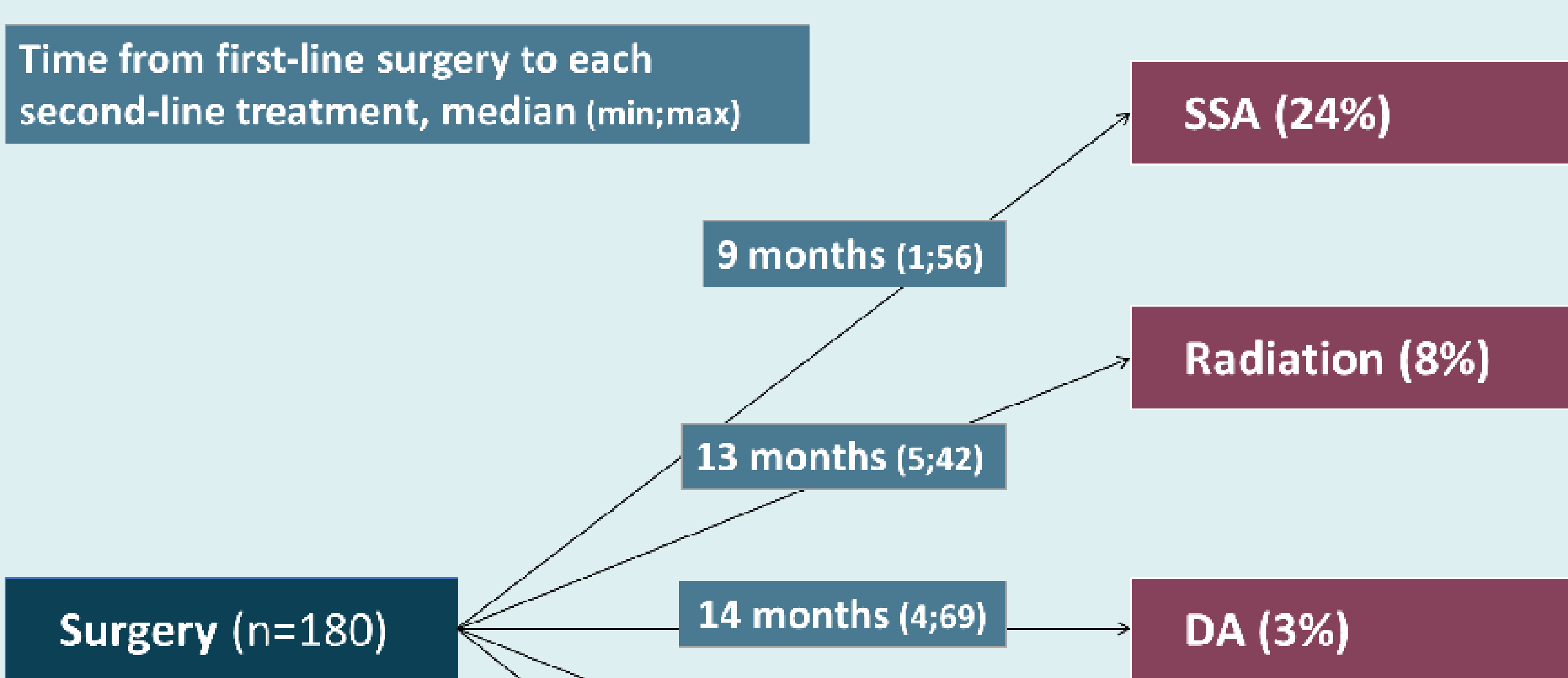

(N/A)

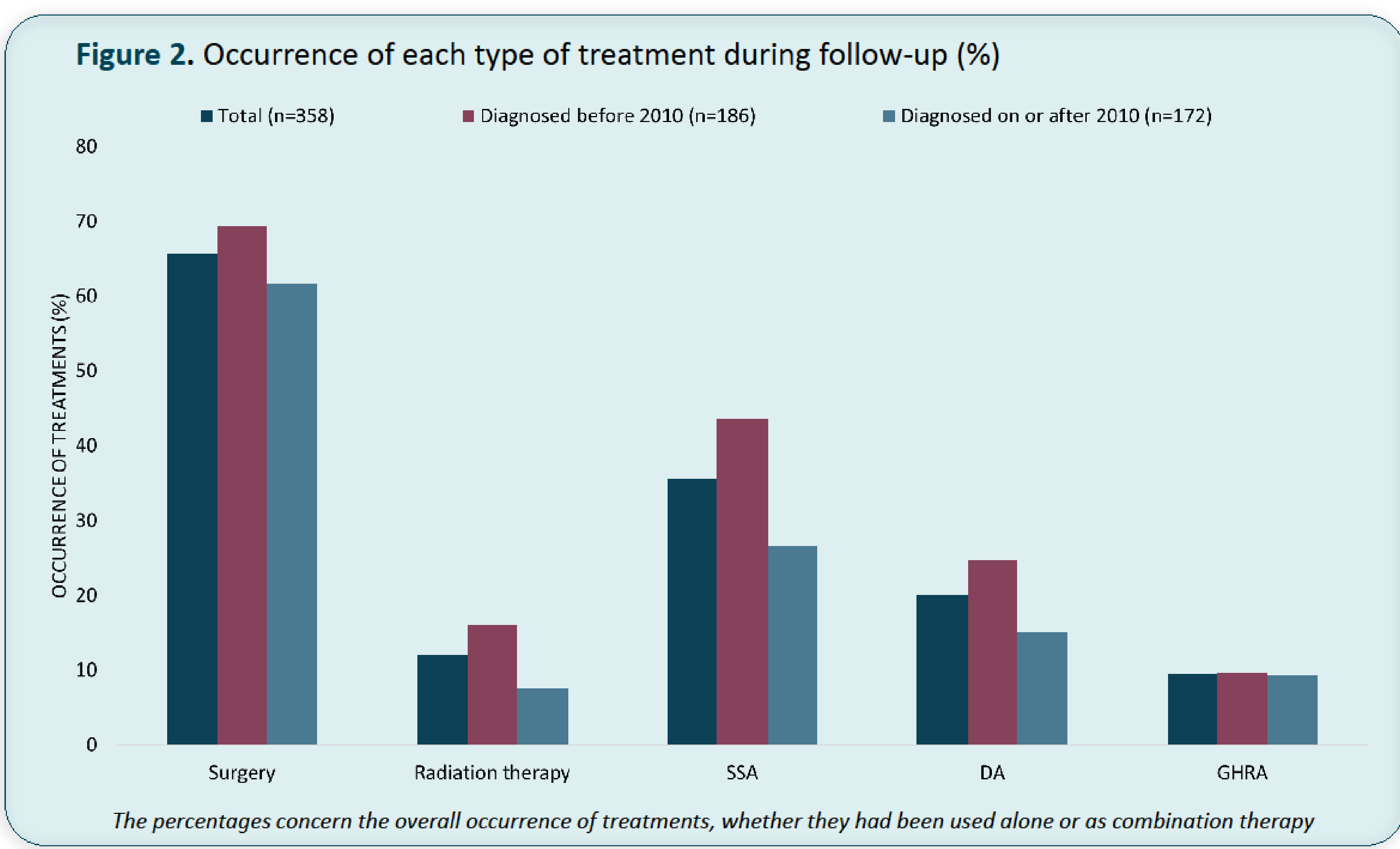

Figure 4. Second-line treatments after first-line SSA, in the total study population

\section{First-line treatment}

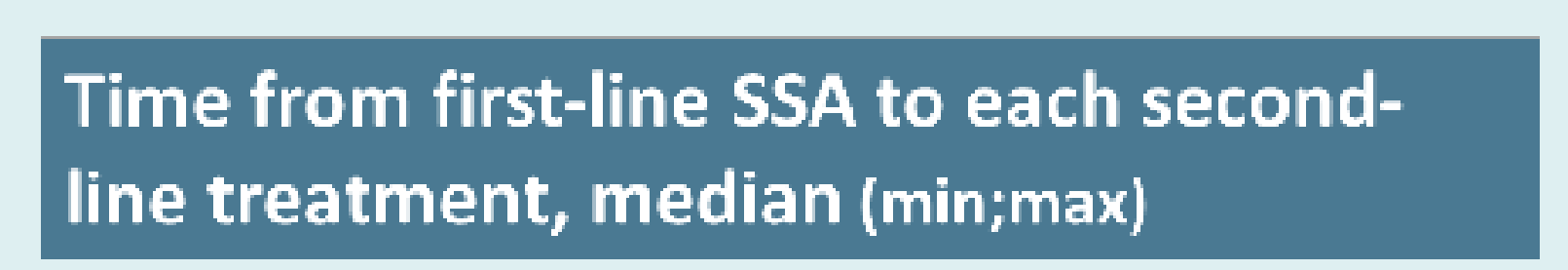

\section{Second-line treatments}

Surgery $(58 \%)$

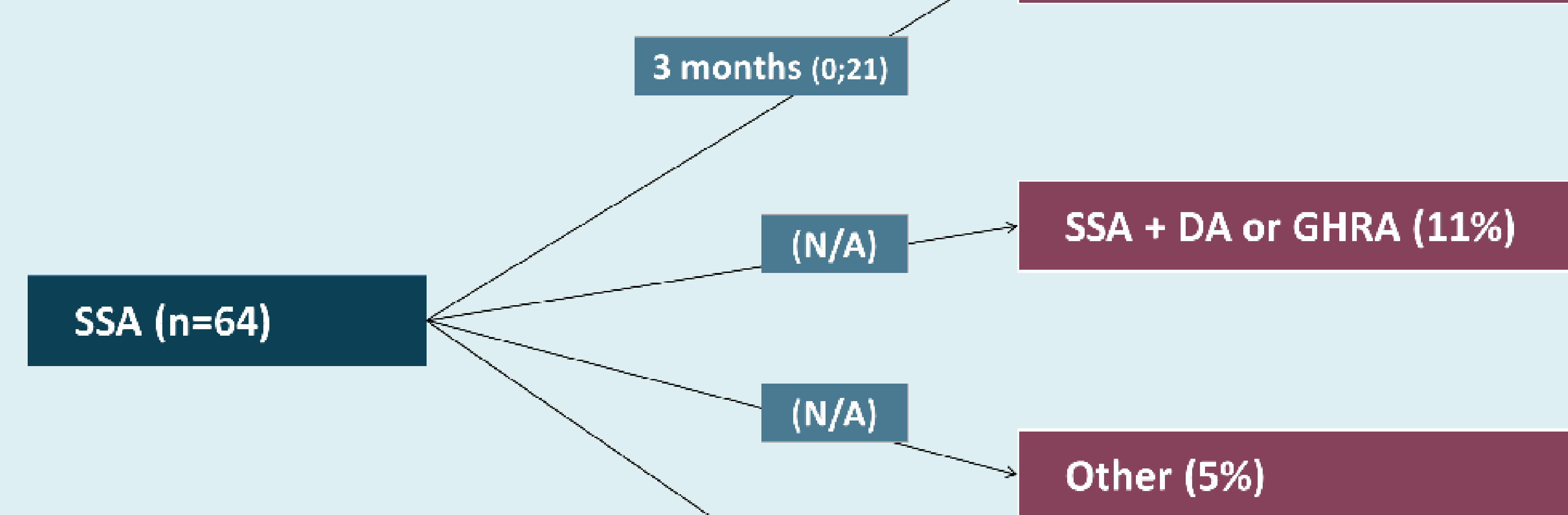

(N/A)
None (27\%)

The total exceeds $100 \%$ due to rounding 